\title{
Relaciones agua/cemento en diseño de vértices extremos aplicado a mortero
}

\author{
William B. Lema ${ }^{1}$ (D), Edison M. Castillo ${ }^{2}$ \\ ${ }^{1}$ Investigador Independiente, Cuenca, Ecuador. \\ ${ }^{2}$ Facultad de Arquitectura y Urbanismo, Universidad de Cuenca, Av. 12 de abril s/n, Cuenca, \\ Ecuador.
}

Autor para la correspondencia: william.lemata@hotmail.com

Fecha de recepción: 22 de noviembre 2017 - Fecha de aceptación: 15 de abril 2018

\section{RESUMEN}

La presente investigación trata de la aplicación de las relaciones agua/cemento en el diseño de mezclas de vértices extremos para morteros, con el fin de delimitar una zona de operabilidad en el simplex. Tres componentes fueron usados, agua, cemento Portland puzolánico y arena fina, las respuestas a resistencias a la compresión a 24 h, 3, 7 y 28 días, y flujo de morteros. El análisis realizado mostró que las relaciones agua/cemento pueden ser modeladas bajo los principios de diseño de mezclas, para luego ser usadas como restricciones para la aplicación del diseño de vértices extremos. Los resultados demostraron que las relaciones agua/cemento tienen rangos delimitadores. Los modelos matemáticos ajustados explican las respuestas con $R_{a j}{ }^{2}$ mayores a $85 \%$ y con valores $p<0.009$. Con todo esto se logró capturar una zona de operabilidad, factible en el simplex para morteros, donde se puede hallar mezclas con relaciones entre $0.3 \leq \mathrm{x}_{1} / \mathrm{x}_{2} \leq 0.9$, con resistencias desde 75.67 hasta $473.35 \mathrm{~kg} / \mathrm{cm}^{2}$ para 28 días y flujos desde 45.15 hasta $150 \%$. dicha zona de factibilidad muestra costos de los morteros por $1 \mathrm{~m}^{3}$ desde 65.94 hasta 229.73\$, que pueden ser tomadas dependiendo de las necesidades técnicas del constructor.

Palabras clave: Diseño de vértices extremos, relación agua/cemento, mortero, modelo matemático.

\begin{abstract}
The present research deals with the application of the water/cement relations in extreme vertices design for mortars to delimit an operability zone in the simplex. Three components were used: water, Portland pozzolanic cement, and fine sand, and the responses were compressive strengths at $24 \mathrm{~h}, 3,7$ and 28 days, and mortar fluidity. The performed analysis revealed that the water/cement relations can be modeled according to the principles of the experimental mix design and they can be used as constraints for the application of the extreme vertices design. Results showed that water/cement ratios have delimited ranges. The mathematical models explain the response with $R_{a j}{ }^{2}$ greater than $85 \%$ and with values of $p<0.009$. In addition, results proved the existence of a feasible operability zone in the simplex for mortars of which the ratio water/cement varies in the range $0.3 \leq \mathrm{x}_{1} / \mathrm{x}_{2} \leq 0.9$, with the resistances varying between 75.67 to $473.35 \mathrm{~kg} / \mathrm{cm}^{2}$ at 28 days and the fluidity from 45.15 to $150 \%$. The mortar cost in the operability zone varies from 65.94 to $229.73 \$$ per $^{3}$, enabling the constructor to select the mortar corresponding to the technical needs.
\end{abstract}

$\underline{\text { Keywords: }}$ Extreme vertices design, water/cement relation, mortar, mathematical model.

\section{INTRODUCCIÓN}

El mortero de cemento es una mezcla de cemento, agua y agregado fino o arena, que al endurecerse adopta propiedades químicas, físicas y mecánicas similares al del concreto y es usado en la construcción, con especial importancia en mampostería estructural (Sánchez de Guzmán, 2001). Para realizar mezclas de morteros existen normas como ASTM C270-10, NTE INEN 2518 y libros de tecnología del concreto 
y mortero, como Sánchez de Guzmán (2001), Neville (1999), Rivera (2013). En las normas y libros se indica el proceso de mezclado y se muestran tablas y gráficas, guías de ensayos y pruebas en laboratorios, pero no se brinda un procedimiento o metodología para hallar las fracciones de las mezclas o una zona de factibilidad para el modelado matemático u optimización. Una de las referencias más importantes para trabajar con concreto lo da el Institute of American Construction (ACI) en un documento guía ACI Committee 211 (2002). Este documento presenta dos métodos para realizar mezclas de concreto, pero no dice nada acerca de los morteros, además, no brinda información en el campo del diseño experimental de mezclas. El diseño experimental de mezclas, aunque es muy poco estudiado en el campo del mortero, tiene espacio para el concreto, por ejemplo, Simon, Lagergren, \& Snyder (1997) realizaron un diseño experimental de mezclas con restricciones y lo optimizaron, en función de la resistencia a la compresión y con la finalidad de obtener un concreto de alta resistencia. También, Dinga, Yan, \& Zhu (1999) aplicaron el diseño de vértices extremos en concreto, con la adición de una combinación de minerales, para observar el efecto que tienen, en la resistencia, la compresión. En Yeh (2006), se trata el análisis de la resistencia a la compresión del concreto al adicionar cenizas volantes a la mezcla. En dicha investigación se utiliza un método simplex centroide con un análisis basado en redes neuronales. Una de las investigaciones que da espacio al estudio de morteros es la de Akalin, Akay, Sennaroglu, \& Tez (2010) que trata de una prueba para encontrar una relación máxima entre la resistencia a la compresión y mínimo costo, usando para este objetivo, los principios de diseño de mezclas en mortero y sugiriendo un método gráfico. En general, estas investigaciones utilizan los principios del diseño experimental, aplicándolos al concreto y mortero, y los investigadores buscan observar y relacionar las proporciones de los componentes en la mezcla con la resistencia a la compresión y la manera de optimizarlo. Sin embargo, ninguna de estas investigaciones trata sobre las relaciones agua/cemento en diseños de mezclas o cómo esto se puede modelar en dicha metodología, tampoco tratan de delimitar una zona experimental factible, en función de las relaciones agua/cemento en mortero simple.

La cantidad de agua y cemento para formar una mezcla manejable debe ser precisada y delimitada con un análisis experimental. La interacción entre estos componentes es importante porque durante la interacción de los minerales del cemento con el agua se producen reacciones químicas como la hidrólisis e hidratación, y procesos físicoquímicos que dan lugar a productos hidratados mecánicamente, resistentes y estables (Muñoz \& Grau, 2013). Sin una combinación precisa de estos elementos, una mezcla puede adquirir propiedades diferentes a las esperadas. Los beneficios de tener mezclas con valores precisos también repercuten en costos en la rama de la construcción. Un consumo de cemento excesivo, o de agua y de agregado, desconociendo que existe una gran variedad de combinaciones que pueden dar resultados que pueden minimizar los costos. El diseño experimental de mezclas es de mucho interés debido a que este método no trata de producir las formulaciones que se le ocurran al experimentador, y llevar a cabo una investigación prueba y error, sino de aplicar una metodología de planeación y análisis que asegure obtener conocimiento y soluciones (Gutiérrez \& De la Vara, 2008). Dentro del diseño experimental se encuentra el diseño de vértices extremos, el mismo que sirve cuando los componentes están sujetos a restricciones. Las restricciones están dadas por razones técnicas o económicas en los componentes, para esto, un conocimiento técnico es importante.

En base a lo expuesto se puede generar la siguiente hipótesis: utilizando las relaciones agua/cemento en el diseño de vértices extremos se puede delimitar una región experimental de operabilidad a una zona del simplex, para obtener mezclas para morteros. Para probar esta hipótesis se plantea los siguientes objetivos: i) delimitar una región experimental en base a las relaciones agua/cemento, para aplicarlo en el diseño experimental de vértices extremos; ii) modelar matemáticamente las respuestas de interés, en función de las proporciones de los componentes de la mezcla; y iii) usar dichos modelos para determinar en qué porcentaje debe participar cada uno de los ingredientes para lograr que la mezcla tenga las propiedades deseadas, análisis de costos.

\section{MATERIALES Y MÉTODOS}

\subsection{Componentes de las mezclas y variables de respuestas}

MASKANA, Vol. 9, No. 1, 125-140, 2018

https://publicaciones.ucuenca.edu.ec/ojs/index.php/maskana/article/view/1860 
Los componentes para la mezcla de morteros fueron tres: $\mathrm{x}_{1}=$ agua, $\mathrm{x}_{2}=$ cemento Portland puzolánico que cumple con NTE INEN $490 \mathrm{y}$ cuya densidad real es $2.91 \mathrm{~g} / \mathrm{cm}^{3}, \mathrm{y} \mathrm{x}_{3}=$ árido fino pasado por malla 8 o arena cuya granulometría se muestra en la Tabla 1. Las respuestas fueron $\mathrm{y}_{1}=$ resistencia a la compresión a las 24 horas, $\mathrm{y}_{2}=$ resistencia a la compresión a los 3 días, $\mathrm{y}_{3}=$ resistencia a la compresión a los a los 7 días, $\mathrm{y}_{4}=$ resistencia a la compresión a los 28 días, $\mathrm{y}_{5}=$ flujo de mortero.

Tabla 1. Granulometría de la arena utilizada en los experimentos.

\begin{tabular}{cccc}
\hline Abertura $(\mathrm{mm})$ & Peso retenido & \% Retenido & \% Acumulado \\
\hline 4.75 & 0.00 & 0.00 & 0.00 \\
2.36 & 0.00 & 0.00 & 0.00 \\
1.18 & 7.50 & 1.50 & 1.50 \\
0.85 & 173.20 & 34.65 & 36.15 \\
0.60 & 258.90 & 51.80 & 87.96 \\
0.30 & 56.20 & 11.24 & 99.20 \\
0.15 & 3.10 & 0.62 & 99.82 \\
0.08 & 0.40 & 0.08 & 99.90 \\
$<$ & 0.50 & 0.10 & 100.00 \\
& 499.80 & 100.00 & \\
\hline D60 & 0.82 & $\mathrm{Cu}$ & 1.41 \\
D10 & 0.58 & $\mathrm{Cc}$ & 1.03 \\
D30 & 0.70 & $\mathrm{Mf}$ & 3.25 \\
\hline Densidad real & $2.59 \mathrm{~g} / \mathrm{cm}^{3}$ & &
\end{tabular}

\subsection{Métodos de ensayos}

Para la mezcla mecánica del mortero se utilizó el método ASTM C305-12, el método ASTM C 349 se utilizó para la caracterización de la resistencia mecánica, y el método ASTM C1437 para medir el flujo de morteros.

\subsection{Diseño experimental de mezclas}

En un experimento con mezclas se tendrá q componentes y cada tratamiento en el experimento corresponde a una combinación específica de estos componentes o mezcla, se denota a las proporciones de los componentes de la mezcla como $\mathrm{x}_{1}, \mathrm{x}_{2}, \mathrm{x}_{3}, \ldots . \mathrm{x}_{\mathrm{q}}$; estas deben cumplir las siguientes condiciones (Gutiérrez \& De la Vara, 2008):

$$
\begin{gathered}
\sum_{i=1}^{\mathrm{q}} \mathrm{x}_{\mathrm{i}}=\mathrm{x}_{1}+\mathrm{x}_{2}+\mathrm{x}_{3} \ldots+\mathrm{x}_{\mathrm{q}}=1 \\
0 \leq \mathrm{x}_{\mathrm{i}} \leq 1, \text { para } \mathrm{i}=1,2,3 \ldots \mathrm{q}
\end{gathered}
$$

En los diseños de mezclas sus puntos experimentales se representan mediante simplejos, que son figuras geométricas que permite graficar q componentes en (q-1) dimensiones. En diseño de mezclas existen varios modelos estadísticos para hallar los puntos experimentales, uno de ellos, o el más básico, es el modelo simplex reticular, simplex centroide y al simplex aumentado (Gutiérrez \& De la Vara, 2008). Los diseños de mezclas con restricciones son utilizados cuando, por razones técnicas o económicas, las fracciones de los componentes de las mezclas se encuentran restringidas a ciertos límites de la siguiente manera:

$$
0 \leq \mathrm{a}_{\mathrm{i}} \leq \mathrm{x}_{\mathrm{i}} \leq \mathrm{b}_{\mathrm{i}} \leq 1
$$

donde $a_{i}$ es el límite inferior de proporción del i-esimo componente $x$ y $b_{i}$ es su límite superior de proporción del i-esimo componente $\mathrm{x}$; en todas las mezclas a probar deben cumplirse estas restricciones a los componentes. Estas restricciones describen un poliedro irregular en una subregión del simplex de (q-1) dimensiones, el centroide y los vértices de este describen un conjunto único de puntos experimentales, los cuales pueden ser utilizados para hallar la superficie de respuesta. 


\section{Diseño de vértices extremos}

El diseño de vértices extremos se basa en encontrar los vértices definidos por las restricciones de los componentes de la mezcla, tomando en cuenta que los vértices deben caer en el límite formado por las restricciones superiores e inferiores de los (q-1) componentes. Según McLean \& Anderson (1966) los vértices son hallados mediante de las siguientes reglas:

- Escribir todas las posibles combinaciones usando los niveles superior e inferior de los componentes, dejando un espacio en blanco de un componente, ejemplo $\left(\mathrm{a}_{1}, \mathrm{~b}_{2}, \ldots, \mathrm{d}_{4}\right)$ para cuatro componentes, para encontrar el número de combinaciones posibles se utiliza la ecuación 2, incluyendo el componente en blanco.

$$
\text { posibles tratamientos }=\mathrm{q} * 2^{\mathrm{q}-1}
$$

- Llenar todos los espacios en blanco de todos los posibles tratamientos encontrados, de manera que cumplan con la restricción de la ecuación 1 y también las restricciones de los niveles superiores e inferiores, obtenidas con anticipación, al final cada una de las combinaciones admitidas es un vértice, pero estos pueden aparecer más de una vez, así que hay que eliminar los que se repiten.

\section{$\underline{\text { Relaciones agua/cemento en diseño de mezclas }}$}

Para incluir las relaciones agua/cemento en el diseño de mezclas se realizó el siguiente procedimiento:

$$
\frac{x_{1}}{x_{2}}=r
$$

Despejando $\mathrm{x}_{1}$ de 3 para remplazarlo en $1 \mathrm{y}$ despejando $\mathrm{x}_{3}$ se tiene.

$$
\mathrm{x}_{3}=1-\mathrm{x}_{2}(1+\mathrm{r})
$$

donde $\mathrm{r}$ se halla como una progresión aritmética dentro de un intervalo $0<\mathrm{r}, \mathrm{r}+\mathrm{d}, \mathrm{r}+2 \mathrm{~d}, \ldots, \mathrm{r}+(\mathrm{n}-1) \mathrm{d}<1$ y donde d toma un valor de incremento pequeño dentro del intervalo. Estos valores se remplazan en 4 , se obtienen ecuaciones en función de $\mathrm{x}_{2}$, los cortes con el eje $\mathrm{x}_{2}$ dan intervalos abiertos para este como sigue.

$\mathrm{x}_{2}$ toma los siguientes rangos $\left(0, \frac{1}{(1+\mathrm{r})}\right) ;\left(0, \frac{1}{(1+\mathrm{r}+\mathrm{d})}\right) ; \ldots ;\left(0, \frac{1}{(1+\mathrm{r}+(\mathrm{n}-1) \mathrm{d})}\right)$

En cada intervalo se halla $\mathrm{x}_{2}$ con una progresión aritmética $\mathrm{x}_{2}, \mathrm{x}_{2}+\mathrm{d}, \mathrm{x}_{2}+2 \mathrm{~d}, \ldots, \mathrm{x}_{2}+(\mathrm{n}-1) \mathrm{d}$, donde $0<\mathrm{d}<1$, mientras que $\mathrm{x}_{1}$ se obtiene despejándolo de la ecuación $3 \mathrm{y} \mathrm{x}_{3}$ de la ecuación 4 . La cantidad de experimentos está en función de las relaciones $\mathrm{r}$ y del incremento pequeño d que se tome, así también de $\mathrm{x}_{2}$, en cada intervalo, los experimentos que se hallen marcarán un área experimental en el simplex.

\section{Modelos matemáticos}

Los modelos matemáticos usados para la interpretación de las respuestas en diseño de mezclas son los siguientes:

- Modelo canónico de primer orden o modelo lineal:

$$
\hat{y}=\sum_{i=1}^{q} \beta_{i} x_{i}
$$

- Modelo canónico de segundo orden o modelo cuadrático:

$$
\hat{y}=\sum_{i=1}^{q} \beta_{i} x_{i}+\sum_{i<j} \sum_{j=2}^{q} \beta_{i j} x_{i} x_{j}
$$

- Modelo canónico de tercer orden o modelo cubico especial: 


$$
\hat{y}=\sum_{i=1}^{q} \beta_{i} x_{i}+\sum_{i<j} \sum_{j=2}^{q} \beta_{i j} x_{i} x_{j}+\sum_{i<j} \sum_{j<k} \sum_{k=3}^{q} \beta_{i j k} x_{i} x_{j} x_{k}
$$

○ Modelo cúbico completo:

$$
\hat{y}=\sum_{i=1}^{q} \beta_{i} x_{i}+\sum_{i<j} \sum_{j=2}^{q} \beta_{i j} x_{i} x_{j}+\sum_{i<j} \sum_{j=2}^{q} \delta_{i j} x_{i} x_{j}\left(x_{i}-x_{j}\right)+\sum_{i<j} \sum_{j<k} \sum_{k=3}^{q} \beta_{i j k} x_{i} x_{j} x_{k}
$$

\section{ANÁLISIS Y RESULTADOS}

\subsection{Relaciones agua/cemento en diseño de mezclas}

Para hallar los experimentos con las relaciones $\mathrm{x}_{1} / \mathrm{x}_{2}$ se realizó para $\mathrm{r}=0.1 \mathrm{y} \mathrm{d}=0.1($ Tabla 2$)$.

Tabla 2. Relaciones r, ecuaciones y número de experimentos.

\begin{tabular}{cccccc}
\hline $\mathrm{N}^{\mathrm{ro}}$ & $\mathrm{r}$ & $\mathrm{x}_{1}$ & $\mathrm{x}_{2}$ & $\mathrm{x}_{3}$ & Nro. de experimentos \\
\hline 1 & 0.1 & $0.1 \mathrm{x}_{2}$ & $(0,0.909)$ & $1-1.1 \mathrm{x}_{2}$ & 9 \\
2 & 0.2 & $0.2 \mathrm{x}_{2}$ & $(0,0.833)$ & $1-1.2 \mathrm{x}_{2}$ & 7 \\
3 & 0.3 & $0.3 \mathrm{x}_{2}$ & $(0,0.769)$ & $1-1.3 \mathrm{x}_{2}$ & 7 \\
4 & 0.4 & $0.4 \mathrm{x}_{2}$ & $(0,0.714)$ & $1-1.4 \mathrm{x}_{2}$ & 6 \\
5 & 0.5 & $0.5 \mathrm{x}_{2}$ & $(0,0.667)$ & $1-1.5 \mathrm{x}_{2}$ & 6 \\
6 & 0.6 & $0.6 \mathrm{x}_{2}$ & $(0,0.625)$ & $1-1.6 \mathrm{x}_{2}$ & 5 \\
7 & 0.7 & $0.7 \mathrm{x}_{2}$ & $(0,0.588)$ & $1-1.7 \mathrm{x}_{2}$ & 5 \\
8 & 0.8 & $0.8 \mathrm{x}_{2}$ & $(0,0.556)$ & $1-1.8 \mathrm{x}_{2}$ & 5 \\
\hline
\end{tabular}

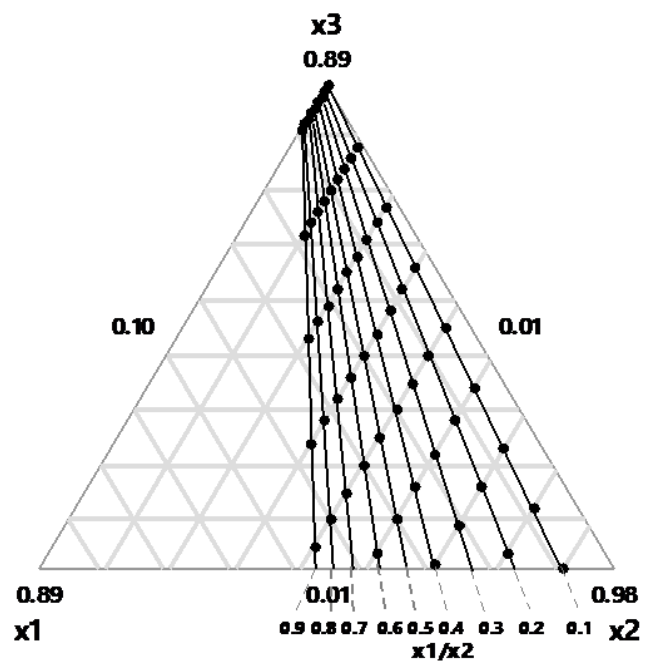

Figura 1. Relaciones $\mathrm{x}_{1} / \mathrm{x}_{2}$ en el simplex para morteros.

Para $\mathrm{x}_{2}=0.1 \mathrm{y} \mathrm{d}=0.1$ se hallan un total de 58 puntos experimentales, como se muestra en la Figura 1. Los experimentos son los puntos en el simplex que se encuentran en las líneas de $\mathrm{x}_{1} / \mathrm{x}_{2}$, que van desde 0.1 hasta 0.9 , marcando una primera área experimental, en dicha área experimental hay que analizar una posible zona con un gran potencial de factibilidad. Así, al observar las relaciones bajas como 0.1 y 0.2 , se puede notar que sus mezclas contienen proporciones de cemento muy altas, y bajas proporciones de agua, lo que evita la formación de mezclas plásticas. Por lo tanto, se desechan estas relaciones de los experimentos, y los experimentos con proporciones bajas de $\mathrm{x}_{1} \mathrm{y} \mathrm{x}_{2}$, así como también las muy altas, no darán mezclas plásticas para relaciones entre 0.3 y 0.9 , por lo que se decidió aplicar un test de flujo en las regiones experimentales medias para cada relación (Tabla 3). 
Los valores cero en la Tabla 3 indican mezclas secas o que no pueden realizarse porque no existe una relación agua/cemento adecuada para llegar a un estado plástico y manejable, por lo tanto, no poseen flujo, los valores mayores a $155 \%$ de flujo indican dónde las mezclas dejan de tener cierta consistencia, desistiendo del estado plástico y pasando a un estado totalmente fluido.

Tabla 3. Experimentos con relaciones $\mathrm{x}_{1} / \mathrm{x}_{2} \mathrm{y}$ flujo de morteros.

\begin{tabular}{|c|c|c|c|c|c|c|}
\hline Nro. Experimento & $\mathrm{x}_{1}$ & $\mathrm{x}_{2}$ & $\mathrm{x}_{3}$ & Suma & $\mathrm{y}=\mathrm{x}_{1} / \mathrm{x}_{2}$ & Test de flujo (\%) \\
\hline 21 & 0.12 & 0.40 & 0.48 & 1 & \multirow{4}{*}{0.3} & 0.00 \\
\hline 22 & 0.15 & 0.50 & 0.35 & 1 & & 0.00 \\
\hline 23 & 0.18 & 0.60 & 0.22 & 1 & & 45.15 \\
\hline 24 & 0.21 & 0.70 & 0.09 & 1 & & 55.25 \\
\hline 27 & 0.12 & 0.30 & 0.58 & 1 & \multirow{5}{*}{0.4} & 0.00 \\
\hline 28 & 0.16 & 0.40 & 0.44 & 1 & & 58.73 \\
\hline 29 & 0.20 & 0.50 & 0.30 & 1 & & 119.28 \\
\hline 30 & 0.24 & 0.60 & 0.16 & 1 & & 148.40 \\
\hline 31 & 0.28 & 0.70 & 0.02 & 1 & & 149.00 \\
\hline 34 & 0.15 & 0.30 & 0.55 & 1 & \multirow{4}{*}{0.5} & 42.70 \\
\hline 35 & 0.20 & 0.40 & 0.40 & 1 & & 143.50 \\
\hline 36 & 0.25 & 0.50 & 0.25 & 1 & & $>155$ \\
\hline 37 & 0.30 & 0.60 & 0.10 & 1 & & $>155$ \\
\hline 39 & 0.12 & 0.20 & 0.68 & 1 & \multirow{4}{*}{0.6} & 0.00 \\
\hline 40 & 0.18 & 0.30 & 0.52 & 1 & & 150.00 \\
\hline 41 & 0.24 & 0.40 & 0.36 & 1 & & $>155$ \\
\hline 42 & 0.30 & 0.50 & 0.20 & 1 & & $>155$ \\
\hline 45 & 0.14 & 0.20 & 0.66 & 1 & \multirow{3}{*}{0.7} & 28.08 \\
\hline 46 & 0.21 & 0.30 & 0.49 & 1 & & $>155$ \\
\hline 47 & 0.28 & 0.40 & 0.32 & 1 & & $>155$ \\
\hline 50 & 0.16 & 0.20 & 0.64 & 1 & \multirow{3}{*}{0.8} & 78.60 \\
\hline 51 & 0.24 & 0.30 & 0.46 & 1 & & $>155$ \\
\hline 52 & 0.32 & 0.40 & 0.28 & 1 & & $>155$ \\
\hline 55 & 0.18 & 0.20 & 0.62 & 1 & \multirow{3}{*}{0.9} & 150.50 \\
\hline 56 & 0.27 & 0.30 & 0.43 & 1 & & $>155$ \\
\hline 57 & 0.36 & 0.40 & 0.24 & 1 & & $>155$ \\
\hline
\end{tabular}

En cada relación de $\mathrm{x}_{1} / \mathrm{x}_{2}$ hay extremos donde los valores de flujos marcan límites, también se observa que, a pesar de tener las mismas relaciones $\mathrm{x}_{1} / \mathrm{x}_{2}$, los conjuntos de experimentos poseen comportamientos diferentes en la respuesta o flujo, en la mayoría de los resultados. En base a este análisis, y con la finalidad de hallar una zona experimental factible, se halla las restricciones para el diseño de vértices extremos como sigue:

$$
\begin{aligned}
& 0.15 \leq \mathrm{x}_{1} \leq 0.24 \\
& 0.20 \leq \mathrm{x}_{2} \leq 0.60 \\
& 0.16 \leq \mathrm{x}_{3} \leq 0.64
\end{aligned}
$$

\subsection{Diseño de vértices extremos}

En la Tabla 4 se muestra los experimentos usando el algoritmo de vértices extremos.

Tabla 4. Experimentos obtenidos por el método de vértices extremos.

\begin{tabular}{cccccc}
\hline $\mathrm{N}^{0}$ experimento & $\mathrm{x}_{1}$ & $\mathrm{x}_{2}$ & $\mathrm{x}_{3}$ & Suma & $\mathrm{x}_{1} / \mathrm{x}_{2}=\mathrm{r}$ \\
\hline 1 & 0.15 & 0.60 & 0.25 & 1 & 0.25 \\
2 & 0.24 & 0.20 & 0.56 & 1 & 1.20 \\
3 & 0.24 & 0.60 & 0.16 & 1 & 0.40 \\
4 & 0.16 & 0.20 & 0.64 & 1 & 0.80 \\
5 & 0.15 & 0.21 & 0.64 & 1 & 0.71 \\
6 & 0.20 & 0.35 & 0.45 & 1 & 0.59 \\
\hline
\end{tabular}


Con la obtención de los experimentos se delimita una nueva área experimental, como se muestra en la Figura 2, el polígono formado son los puntos experimentales obtenidos con el método de vértices extremos; cada vértice del polígono formado es un experimento, así como el centroide. Se muestran en esta figura también las relaciones $\mathrm{x}_{1} / \mathrm{x}_{2}$, desde 0.3 hasta 0.9. Como se puede observar en la Figura 2 , existen experimentos dentro del polígono como resultado de las relaciones $\mathrm{x}_{1} / \mathrm{x}_{2}$, que pueden ser utilizados de forma adicional.

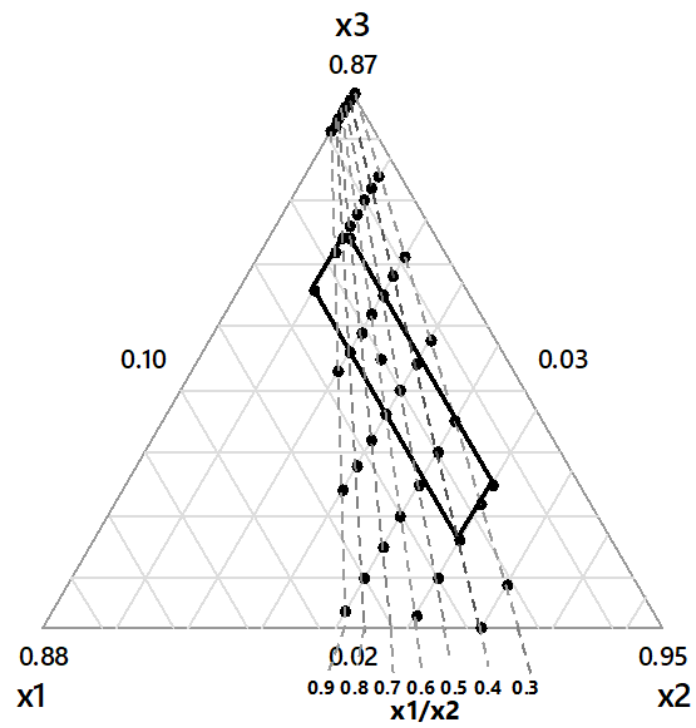

Figura 2. Vértices extremos y relaciones $\mathrm{x}_{1} / \mathrm{x}_{2}$.

\subsection{Experimentos}

Se procede a seleccionar de maneara aleatoria los experimentos de la Tabla 3 que cumplan con las restricciones y unirla a la Tabla 4 para obtener la Tabla 5.

Tabla 5. Experimentos y relaciones $\mathrm{x}_{1} / \mathrm{x}_{2}$.

\begin{tabular}{cccccc}
\hline $\mathrm{N}^{0}$ experimento & $\mathrm{x}_{1}$ & $\mathrm{x}_{2}$ & $\mathrm{x}_{3}$ & Suma & $\mathrm{x}_{1} / \mathrm{x}_{2}$ \\
\hline 1 & 0.15 & 0.60 & 0.25 & 1 & 0.25 \\
2 & 0.24 & 0.20 & 0.56 & 1 & 1.20 \\
3 & 0.24 & 0.60 & 0.16 & 1 & 0.40 \\
4 & 0.16 & 0.20 & 0.64 & 1 & 0.80 \\
5 & 0.15 & 0.21 & 0.64 & 1 & 0.71 \\
6 & 0.20 & 0.35 & 0.45 & 1 & 0.59 \\
7 & 0.18 & 0.60 & 0.22 & 1 & 0.30 \\
8 & 0.16 & 0.40 & 0.44 & 1 & 0.40 \\
9 & 0.20 & 0.50 & 0.30 & 1 & 0.50 \\
10 & 0.15 & 0.30 & 0.55 & 1 & 0.50 \\
11 & 0.20 & 0.40 & 0.40 & 1 & 0.60 \\
13 & 0.18 & 0.30 & 0.52 & 1 & 0.90 \\
\hline
\end{tabular}

\subsection{Resultados de los experimentos}

Los resultados promedios de tres réplicas se muestran en la Tabla 6, el orden experimental fue aleatorio pero la tabla se ordenó de mayor a menor, en función de las relaciones $\mathrm{x}_{1} / \mathrm{x}_{2}$. 
Tabla 6. Resultados de los experimentos.

\begin{tabular}{cccccccccc}
\hline Experimento & $\mathrm{x}_{1}$ & $\mathrm{x}_{2}$ & $\mathrm{x}_{3}$ & $\mathrm{x}_{1} / \mathrm{x}_{2}$ & $\mathrm{y}_{1}\left(\mathrm{~kg} / \mathrm{cm}^{2}\right)$ & $\mathrm{y}_{2}\left(\mathrm{~kg} / \mathrm{cm}^{2}\right)$ & $\mathrm{y}_{3}\left(\mathrm{~kg} / \mathrm{cm}^{2}\right)$ & $\mathrm{y}_{4}\left(\mathrm{~kg} / \mathrm{cm}^{2}\right)$ & $\mathrm{y}_{5}$ test flujo $(\%)$ \\
\hline 1 & 0.15 & 0.60 & 0.25 & 0.25 & 159.43 & 253.77 & 380.57 & 450.11 & 0.00 \\
7 & 0.18 & 0.60 & 0.22 & 0.30 & 141.53 & 299.87 & 430.83 & 473.35 & 45.15 \\
8 & 0.16 & 0.40 & 0.44 & 0.40 & 61.27 & 251.90 & 309.80 & 438.90 & 58.73 \\
9 & 0.20 & 0.50 & 0.30 & 0.40 & 40.93 & 192.38 & 215.93 & 440.17 & 119.28 \\
3 & 0.24 & 0.60 & 0.16 & 0.40 & 89.03 & 241.97 & 299.20 & 411.60 & 148.40 \\
10 & 0.15 & 0.30 & 0.55 & 0.50 & 42.67 & 126.73 & 200.47 & 306.67 & 42.70 \\
11 & 0.20 & 0.40 & 0.40 & 0.50 & 50.10 & 116.47 & 193.50 & 287.73 & 143.50 \\
6 & 0.20 & 0.35 & 0.45 & 0.59 & 13.47 & 49.17 & 125.60 & 243.77 & 155.00 \\
12 & 0.18 & 0.30 & 0.52 & 0.60 & 25.37 & 80.13 & 121.80 & 217.77 & 150.00 \\
5 & 0.15 & 0.21 & 0.64 & 0.71 & 19.63 & 46.83 & 70.40 & 124.97 & 44.93 \\
4 & 0.16 & 0.20 & 0.64 & 0.80 & 16.00 & 39.40 & 60.23 & 106.10 & 78.60 \\
13 & 0.18 & 0.20 & 0.62 & 0.90 & 12.17 & 24.60 & 40.97 & 75.67 & 150.50 \\
2 & 0.24 & 0.20 & 0.56 & 1.20 & 0.00 & 0.00 & 0.00 & 0.00 & $>155.00$ \\
\hline
\end{tabular}

De la Tabla 6 se observa que el porcentaje de incremento promedio de la resistencia de los experimentos de $24 \mathrm{~h}$ a los 3 días es $185 \%$, el porcentaje de incremento promedio de 3 días, a los 7 días, es de $54.50 \%$, y a los 28 días es de $60.34 \%$. En las relaciones bajas de $\mathrm{x}_{1} / \mathrm{x}_{2}$, entre 0.25 a 0.3 , los valores de resistencia a la compresión son altos, pero el incremento al pasar el tiempo parece no ser alto, con un promedio de $48.8 \%$; el incremento de la resistencia a la compresión en relaciones de $\mathrm{x}_{1} / \mathrm{x}_{2}$, entre 0.4 y 0.9 , está en un promedio de $110 \%$. De la Tabla 6 también se observa que, a medida que aumentan las relaciones $\mathrm{x}_{1} / \mathrm{x}_{2}$, parece existir una tendencia a disminuir la resistencia a la compresión en la mayoría de los experimentos. Cada experimento con su relación respectiva tiene resultados diferentes, aunque tenga la misma relación $\mathrm{x}_{1} / \mathrm{x}_{2}$, como se puede observar en los experimentos con relaciones de 0.4 y 0.5 . El experimento 1 , con relación $\mathrm{x}_{1} / \mathrm{x}_{2}=0.25$, no posee resultado de flujo ya que su mezcla es seca, por otro lado, las relaciones de 0.52 y 0.9 , tales como los experimentos 6 y 13 , tienen flujos muy altos, es decir, son mezclas muy fluidas. El experimento 2 , con $\mathrm{x}_{1} / \mathrm{x}_{2}=1.20$, no posee resistencia a la compresión ya que es imposible realizar una mezcla con una proporción mucho mayor de agua que de cemento; esta mezcla no es trabajable y este experimento es parte del diseño de vértices extremos por lo que no se puede eliminar de forma discriminada de la Tabla 6. Este análisis marca dos condiciones más que deben cumplir las mezclas para mortero dentro del diseño de mezclas:

$$
\begin{gathered}
\mathrm{x}_{1}<\mathrm{x}_{2} \\
0.3 \leq \mathrm{x}_{1} / \mathrm{x}_{2} \leq 0.9
\end{gathered}
$$

\section{5. $\quad$ Ajuste de modelos y caracterización de la superficie de respuesta}

A partir de los resultados obtenidos en la Tabla 6 se procede a realizar el análisis de los posibles modelos para la resistencia compresión a 24 h, 3, 7 y 28 días, y flujo de morteros, respectivamente, como se muestra en la Tabla 7. En esta tabla se ha resumido los parámetros más importantes de un ANOVA con la finalidad de ser más perecederos con los resultados.

\section{Resistencia a la compresión a las $24 \mathrm{~h}$}

El análisis para la resistencia a la compresión se encuentra en la Tabla 7, el aporte del modelo lineal es muy significativo, con un $p<0.001$, así también, el aporte del modelo cuadrático es significativo con $p=0.076$, al observar el $p$ del modelo cúbico, este no posee un aporte significativo.

El coeficiente de determinación múltiple $\mathrm{R}^{2}$ del modelo cuadrático es igual al del modelo cúbico, a pesar de que el modelo cúbico tiene un coeficiente más. El valor de $\mathrm{Raj}^{2}$ de este modelo es menor al cuadrático, y la varianza estimada del modelo cúbico es mayor a la del cuadrático. Se puede decir que la adición del coeficiente B123 aporta muy poco a explicar el comportamiento de las respuestas, pero no porque no haya disminuido el error con este modelo, sino porque esa disminución no es lo suficientemente significativa en comparación a los grados de libertad perdidos. Así, se puede tomar el modelo cuadrático o el modelo cúbico, pero de acuerdo con la Tabla 7, el modelo a tomar debe ser el 
cuadrático, por lo tanto, se ajusta dicho modelo y se muestra su ANOVA en la Tabla 8, con su respectiva ecuación 9, que se halla en la Tabla 9.

Tabla 7. Análisis de los posibles modelos para la resistencia a la compresión a las 24 h, 3, 7 y 28 días y flujo de mortero respectivamente.

\begin{tabular}{|c|c|c|c|c|}
\hline Modelos & $\sigma^{2}$ & $\mathrm{R}^{2}$ & $\mathrm{Raj}^{2}$ & $p$ \\
\hline \multicolumn{5}{|l|}{24 horas } \\
\hline Lineal & 450.34 & 0.87 & 0.82 & $<0.001$ \\
\hline Cuadrático & 197.37 & 0.96 & 0.92 & 0.076 \\
\hline Cúbico & 213.94 & 0.96 & 0.91 & $>0.1$ \\
\hline \multicolumn{5}{|l|}{3 días } \\
\hline Lineal & 1521.09 & 0.89 & 0.86 & 0.0027 \\
\hline Cuadrático & 2108.85 & 0.90 & 0.80 & $>0.1$ \\
\hline Cúbico & 1305.23 & 0.95 & 0.88 & 0.0876 \\
\hline \multicolumn{5}{|l|}{7 días } \\
\hline Lineal & 1653.09 & 0.93 & 0.91 & $<0.001$ \\
\hline Cuadrático & 2274.28 & 0.94 & 0.88 & $>0.1$ \\
\hline Cúbico & 1666.20 & 0.96 & 0.91 & $>0.1$ \\
\hline \multicolumn{5}{|l|}{28 días } \\
\hline Lineal & 3659.22 & 0.90 & 0.86 & $<0.001$ \\
\hline Cuadrático & 1558.85 & 0.97 & 0.94 & 0.045 \\
\hline Cúbico & 1253.41 & 0.98 & 0.95 & $>0.1$ \\
\hline \multicolumn{5}{|l|}{ Flujo de mortero } \\
\hline Lineal & 741.61 & 0.83 & 0.80 & $<0.001$ \\
\hline Cuadrático & 322.27 & 0.94 & 0.91 & 0.006 \\
\hline Cúbico & 346.45 & 0.94 & 0.91 & $>0.1$ \\
\hline
\end{tabular}

Tabla 8. Análisis de la varianza de los modelos cuadráticos y cúbicos ajustados para la resistencia a la compresión a 24 h, 3, 7 y 28 días, y flujo de mortero, respectivamente.

\begin{tabular}{|c|c|c|c|c|c|c|}
\hline \multicolumn{2}{|c|}{ Fuente } & Suma de cuadrados & Grados de libertad & Cuadrados medios & $\mathrm{F}$ & $p$ \\
\hline \multirow[t]{4}{*}{$24 \mathrm{~h}$} & Cuadrático & & & & & \\
\hline & SCR & 28871.38 & 6 & 4811.90 & 24.38 & $<0.001$ \\
\hline & SCE & 1184.24 & 6 & 197.37 & & \\
\hline & SCT & 30055.63 & 12 & & & \\
\hline \multirow[t]{4}{*}{3 días } & Cubico & & & & & \\
\hline & SCR & 121197.41 & 7 & 17313.92 & 13.26 & 0.0086 \\
\hline & SCE & 6526.17 & 5 & 1305.23 & & \\
\hline & SCT & 127723.58 & 12 & & & \\
\hline \multirow[t]{4}{*}{7 días } & Cubico & & & & & \\
\hline & SCR & 211276.49 & 7 & 30182.36 & 18.11 & 0.006 \\
\hline & SCE & 8331.00 & 5 & 1666.20 & & \\
\hline & SCT & 219607.49 & 12 & & & \\
\hline \multirow[t]{4}{*}{28 días } & Cuadrático & & & & & \\
\hline & SCR & 305269.25 & 6 & 50878.21 & 32.64 & $<0.001$ \\
\hline & SCE & 9353.12 & 6 & 1558.85 & & \\
\hline & SCT & 314622.36 & 12 & & & \\
\hline \multicolumn{7}{|c|}{ Flujo de mortero Cuadrático } \\
\hline & SCR & 70002.03 & 6 & 11667.00 & 36.20 & $<0.001$ \\
\hline & SCE & 4511.73 & 14 & 322.27 & & \\
\hline & SCT & 74513.76 & 20 & & & \\
\hline
\end{tabular}

De la ecuación 9 el efecto de la interacción de los coeficientes lineales produce un efecto parecido al sinérgico, mientras que los coeficientes cuadráticos producen un efecto antagónico. En la Figura 3(a) se presenta la gráfica de contornos marcada por los vértices extremos y en la Figura 4(a) la superficie de respuesta. El máximo local dentro de dicha zona está en el vértice $(0.15,0.60,0.25)$ con un valor de resistencia estimado de $162.83 \mathrm{~kg} / \mathrm{cm}^{2}$ y una relación $\mathrm{x}_{1} / \mathrm{x}_{2}=0.25$. Las relaciones $\mathrm{x}_{1} / \mathrm{x}_{2}$ bajas, como 0.4 y 0.3 , también tienen resistencias altas, relaciones menores a 0.25 no son realizables ya que las 
proporciones de cemento son exageradas y no forman mezclas plásticas con el agua. Los valores mínimos están cerca del vértice $(0.24,0.20,0.56)$, donde el contorno de 0 y $5 \mathrm{~kg} / \mathrm{cm}^{2}$ prevalecen con una relación $\mathrm{x}_{1} / \mathrm{x}_{2} \geq 0.9$; pero en esta zona es difícil hallar mezclas factibles debido a las altas proporciones de agua, por lo tanto, si se desea minimizar la resistencia se debe interpolar mezclas desde relaciones 0.7 hasta 0.9 , dentro del polígono. En la mitad se encuentran los contornos en sentido creciente hacia el vértice $\mathrm{x}_{2}$. La superficie de respuesta muestra a detalle el comportamiento del modelo en el cual parce existir un valor máximo local en el vértice $\mathrm{x}_{1}$ del simplex, pero este está fuera de la zona experimental y además en esta zona las relaciones $\mathrm{x}_{1} / \mathrm{x}_{2}$ son mayores que 1 , como resultado, es imposible tener mezclas factibles en dicha zona. La zona de valores ceros y negativos en la superficie de respuesta indican dónde los experimentos no se pueden realizar.

Tabla 9. Modelos matemáticos ajustados a la resistencia a la compresión y al flujo de mortero.

\begin{tabular}{rlr}
\hline \multicolumn{1}{c}{ Modelos } & Resistencia \\
\hline $\mathrm{y}_{1}=$ & $2055.04 \mathrm{x}_{1}+839.50 \mathrm{x}_{2}+134.06 \mathrm{x}_{3}-5229.51 \mathrm{x}_{1} \mathrm{x}_{2}-3066.70 \mathrm{x}_{1} \mathrm{x}_{3}-646.57 \mathrm{x}_{2} \mathrm{x}_{3}(9)$ & $24 \mathrm{~h}$ \\
$\mathrm{y}_{2}=$ & $-16671.15 \mathrm{x}_{1}-2042.55 \mathrm{x}_{2}-2674.40 \mathrm{x}_{3}+34139.46 \mathrm{x}_{1} \mathrm{x}_{2}+34538.84 \mathrm{x}_{1} \mathrm{x}_{3}+11343.29 \mathrm{x}_{2} \mathrm{x}_{3}$ & 3 días \\
& $-61756.60 \mathrm{x}_{1} \mathrm{x}_{2} \mathrm{x}_{3}(10)$ & \\
$\mathrm{y}_{3}=$ & $-16244.91 \mathrm{x}_{1}-1486.03 \mathrm{x}_{2}-2581.26 \mathrm{x}_{3}+31477.02 \mathrm{x}_{1} \mathrm{x}_{2}+33248.88 \mathrm{x}_{1} \mathrm{x}_{3}+10688.55 \mathrm{x}_{2} \mathrm{x}_{3}$ & 7 días \\
& $-57517.38 \mathrm{x}_{1} \mathrm{x}_{2} \mathrm{x}_{3}(11)$ & \\
$\mathrm{y}_{4}=$ & $3229.87 \mathrm{x}_{1}+315.90 \mathrm{x}_{2}-229.42 \mathrm{x}_{3}-3588.01 \mathrm{x}_{1} \mathrm{x}_{2}-8153.35 \mathrm{x}_{1} \mathrm{x}_{3}+2553.65 \mathrm{x}_{2} \mathrm{x}_{3}(12)$ & 28 días \\
$\mathrm{y}_{5}=$ & $-4989.91 \mathrm{x}_{1}-1022.28 \mathrm{x}_{2}-430.89 \mathrm{x}_{3}+11260.69 \mathrm{x}_{1} \mathrm{x}_{2}+8962.30 \mathrm{x}_{1} \mathrm{x}_{3}+647.92 \mathrm{x}_{2} \mathrm{x}_{3}(13)$ & Flujo \\
\hline
\end{tabular}

Resistencia a la compresión a los 3 días

Para el ajuste del modelo se realizó el análisis de los posibles modelos, como se muestra en la Tabla 7. En este caso los modelos que aportan de manera significativa son el lineal y el cúbico, con valores de $\mathrm{p}$ muy bajos; al observar los valores de $\mathrm{R}^{2}$ y $\mathrm{Raj}^{2}$, el modelo que mejor resultado muestra es el cúbico. En la Tabla 8 se muestra el ANOVA del modelo cúbico ajustado y su respectiva ecuación 10 en la Tabla 9. Los coeficientes lineales de la ecuación 10 muestran un efecto antagónico, mientras que los coeficientes cuadráticos muestran un efecto sinérgico, y el coeficiente cúbico un efecto antagónico. Para un mayor entendimiento de dicha ecuación se puede observar la Figura 3(b), donde se halla la gráfica de contornos y superficie de respuesta en la Figura 4(b). En los contornos de la Figura 3(b) se observa el incremento de las resistencias en comparación a la Figura 3(a). Aunque la Figura 4(b) los contornos pertenecen al modelo cúbico, se puede observar el incremento del estado endurecido de las mezclas que es hacia el vértice $(0.15,0.60,0.25)$ donde la relación de $\mathrm{x}_{1} / \mathrm{x}_{2}$ es 0.25 , con un máximo de compresión de 284.92 $\mathrm{kg} / \mathrm{cm}^{2}$, hacia el vértice $(0.24,0.20,0.56)$, donde las relaciones están cerca de 1 , está el punto $(0.180 .20$ 0.62 ) donde la relacione es de 0.9 y la resistencia estimada es de $44.2 \mathrm{~kg} / \mathrm{cm}^{2}$, el mismo que sería el punto experimental factible mínimo a los 3 días.

El contorno de valor cero y los valores negativos en la superficie de respuesta indican dónde los experimentos no se pueden realizar. Se puede notar que el modelo cúbico trata de ajustarse muy bien a las respuestas, y de marcar una zona experimental factible, de tal manera que da valores negativos a zonas donde no es posible hallar mezclas factibles.

\section{Resistencia a la compresión a los 7 días}

Para la resistencia a los 7 días se tiene el análisis de los modelos que se muestran en la Tabla7, el aporte de los términos lineales es muy significativo y el de los términos cuadráticos y cúbicos no lo son. Los coeficientes de determinación múltiple muestran los mayores valores para el modelo lineal y cúbico, pero el aporte del modelo cúbico en la regresión debe ser valorado, ya que es mayor que el cuadrático y ayuda a disminuir el error aun cuando sus grados de libertad disminuyen; por lo tanto, en este caso se decide tomar al modelo cúbico. El ANOVA se muestra en la Tabla 8 y su respectiva ecuación 11 en la Tabla 9.

En la Figura 3(c) se muestran las gráficas de contornos y la superficie de respuesta en la Figura 4(c). En la de contornos se muestra los valores de la resistencia a los 7 días, que han aumentado de manera evidente, los contornos de resistencia aumentan a medida que el tiempo pasa con un 
desplazamiento hacia el vértice $(0.15,0.60,0.25)$ del polígono y una disminución hacia el vértice $(0.18$ 0.20 0.62). Al igual que en el caso anterior, los valores negativos de resistencia a la compresión no deben ser interpretados ya que el modelo ajustado solo es una interpretación mediante una relación funcional de los resultados de los experimentos obtenidos, pero estos valores deben ser tomados como una zona donde las mezclas no son factibles.

\section{Resistencia a la compresión a los 28 días}

La selección del modelo para la resistencia a la compresión a los 28 días se halla en la Tabla 7, donde se muestra el aporte de cada modelo con respecto al modelo de orden inferior. Se observa los aportes de cada modelo en la Tabla 7, siendo significativos el aporte lineal y cuadrático, el $p$ del aporte del modelo cúbico no es tan significativo, los valores de $\mathrm{R}^{2}$ y $\mathrm{Raj}^{2}$ son mayores para el modelo cúbico, pero el modelo cuadrático es muy bueno llegando a explicar los resultados tan bien como un modelo cúbico, además, también es más fácil de manejar ya que no contiene otro término. Se presenta el ANOVA del modelo cuadrático en la Tabla 8 y su ecuación 12 en la Tabla 9.

De la ecuación 12 los coeficientes lineales de $\mathrm{x}_{1}$ y $\mathrm{x}_{2}$ presentan efectos sinérgicos, y el coeficiente lineal de $\mathrm{x}_{3}$ un efecto antagónico, mientras los coeficientes cuadráticos aportan efecto antagónico en las interacciones $\mathrm{x}_{1} \mathrm{X}_{2} \mathrm{y} \mathrm{x}_{1} \mathrm{X}_{3}$; el coeficiente de la interacción $\mathrm{x}_{2} \mathrm{X}_{3}$ aporta efecto sinérgico. En la Figura 3(d) se presenta la gráfica de contorno y la superficie de respuesta para el modelo ajustado en la Figura 4(d), en la zona experimental marcada los contornos muestran el incremento de resistencia a la compresión hacia el vértice $(0.15,0.6,0.25)$ con relación $\mathrm{x}_{1} / \mathrm{x}_{2}=0.25$, ahí se halla el máximo local a los 28 días, con una resistencia estimada de $485.7 \mathrm{~kg} / \mathrm{cm}^{2}$; las relaciones 0.3 y 0.4 , con resistencias entre $400 \mathrm{~kg} / \mathrm{cm}^{2}$ y $475 \mathrm{~kg} / \mathrm{cm}^{2}$, están en la zona de máximas resistencias a los 28 días.

Cerca del punto $(0.24,0.20,0.56)$ donde las relaciones $\mathrm{x}_{1} / \mathrm{x}_{2} \geq 1.2$ las resistencias son menores que cero, esto se debe de interpretar como una zona donde las relaciones $\mathrm{x}_{1} / \mathrm{x}_{2}$ son muy altas y las mezclas demasiado fluidas para tener manejabilidad, lo que indica que no se pueden realizar mezclas plásticas en estado fresco con esa relación. Lo mismo ocurre en la relación 1, cerca del contorno de $100 \mathrm{~kg} / \mathrm{cm}^{2}$ es la zona experimental de resistencias mínimas, ahí se hallan los puntos $(0.18,0.20,0.62)$ y $(0.16,0.20$, 0.64), con relaciones 0.9 y 0.8 , las resistencias estimadas que alcanzan son $64.37 \mathrm{~kg} / \mathrm{cm}^{2}$ y $103.9 \mathrm{~kg} / \mathrm{cm}^{2}$, respectivamente, claramente se puede notar que existe una delimitación para las mezclas de morteros. La zona experimental de transición, donde las relaciones van desde 0.7 a 0.4 , muestran valores de resistencia desde 100 a $400 \mathrm{~kg} / \mathrm{cm}^{2}$.

\section{Flujo de morteros}

En el caso del flujo de morteros se usó los experimentos de la Tabla 3, que no fueron añadidos a la Tabla 5 , esto implica experimentos fuera de la zona experimental, como consecuencia, se crea una nueva zona experimental como se verá más adelante, en la Tabla 7 se muestra el análisis para la selección del modelo.

Los modelos lineales y cuadráticos poseen un aporte muy significativo, con valores $\mathrm{p}$ muy pequeños, el aporte cúbico no es significativo, los coeficientes de determinación múltiple muestran una igualdad entre el modelo cuadrático y el cúbico. En este caso el modelo cuadrático es mejor ya que ayuda a explicar los resultados experimentales tanto como lo hace un modelo de orden superior, además, posee una varianza menor. El ANOVA del modelo cuadrático ajustado se muestra en la Tabla 8, en el mismo se aprecia el $p$, que es menor a 0.001 , lo que demuestra que el modelo cuadrático es muy significativo. Los coeficientes lineales de la ecuación 13 producen un efecto parecido al antagónico, mientras los coeficientes cuadráticos un efecto sinérgico, la gráfica de contornos y la superficie de respuesta se muestra en la Figura 3(e) y 4(e).

El comportamiento del flujo en los contornos de la Figura 3(e) muestra un comportamiento inverso a la resistencia a la compresión, además, la zona experimental se amplió, nótese que los puntos experimentales que caen en el contorno de cero están fuera de la zona experimental con respecto a la Figura 3(d), a excepción del punto $(0.15,0.6,0.25)$ con relación $\mathrm{x}_{1} / \mathrm{x}_{2}=0.25$. Este es el máximo local en las resistencias a la compresión, pero la realización de esta mezcla es muy difícil en estado fresco. En la práctica, la proporción alta de cemento provoca una mezcla seca cuyo flujo es inexistente, lo que 
confirma el contorno de 0\% en la Figura 3(e), lo mismo sucede en los otros puntos que se encuentran cerca de este contorno, los mismos que marcan un inicio para las mezclas en cada relación.

En el contorno de $150 \%$ caen experimentos desde el vértice $(0.28,0.7,0.02)$ con relación $\mathrm{x}_{1} / \mathrm{x}_{2}=$ 0.4 hasta el punto $(0.2,0.222,0.577)$ con $\mathrm{x}_{1} / \mathrm{x}_{2}=0.9$, todos los puntos experimentales resultan mezclas muy fluidas lo que indica un final para los experimentos en cada relación. Así, el contorno de $0 \%$ marcaría el límite inferior y el contorno de $150 \%$ marca el límite superior de factibilidad para mezclas con relaciones $0.3 \leq \mathrm{x}_{1} / \mathrm{x}_{2} \leq 0.9$, esta sería la zona de operabilidad para morteros donde los experimentos pueden ser hallados según sus relaciones especificadas con la ecuación 14.

$$
\mathrm{r}=\mathrm{f}\left(\mathrm{x}_{1}, \mathrm{x}_{2}\right)= \begin{cases}\mathrm{x}_{1} / \mathrm{x}_{2}=0.3 & \text { si } 0.15<\mathrm{x}_{1} \leq 0.21 \text { y } 0.5<\mathrm{x}_{2} \leq 0.7 \\ \mathrm{x}_{1} / \mathrm{x}_{2}=0.4 & \text { si } 0.12<\mathrm{x}_{1} \leq 0.28 \text { y } 0.3<\mathrm{x}_{2} \leq 0.7 \\ \mathrm{x}_{1} / \mathrm{x}_{2}=0.5 & \text { si } 0.12<\mathrm{x}_{1} \leq 0.2 \text { y } 0.24<\mathrm{x}_{2} \leq 0.4 \\ \mathrm{x}_{1} / \mathrm{x}_{2}=0.6 & \text { si } 0.12<\mathrm{x}_{1} \leq 0.2 \text { y } 0.2<\mathrm{x}_{2} \leq 0.333 \\ \mathrm{x}_{1} / \mathrm{x}_{2}=0.7 & \text { si } 0.14 \leq \mathrm{x}_{1}<0.20 \text { y } 0.2 \leq \mathrm{x}_{2}<0.285 \\ \mathrm{x}_{1} / \mathrm{x}_{2}=0.8 & \text { si } 0.16 \leq \mathrm{x}_{1}<0.2 \text { y } 0.2 \leq \mathrm{x}_{2}<0.25 \\ \mathrm{x}_{1} / \mathrm{x}_{2}=0.9 & \text { si } 0.18 \leq \mathrm{x}_{1}<0.20 \text { y } 0.2 \leq \mathrm{x}_{2}<0.222\end{cases}
$$

La ecuación 14 permite hallar más experimentos en zonas no exploradas dentro de la zona de operabilidad y los resultados pueden ser estimados con los modelos ajustados. En la Figura 5 se muestra la zona de interés capturada, o la zona de operabilidad obtenida en este análisis, las líneas representan las relaciones $\mathrm{x}_{1} / \mathrm{x}_{2}$ dentro de la zona capturada.

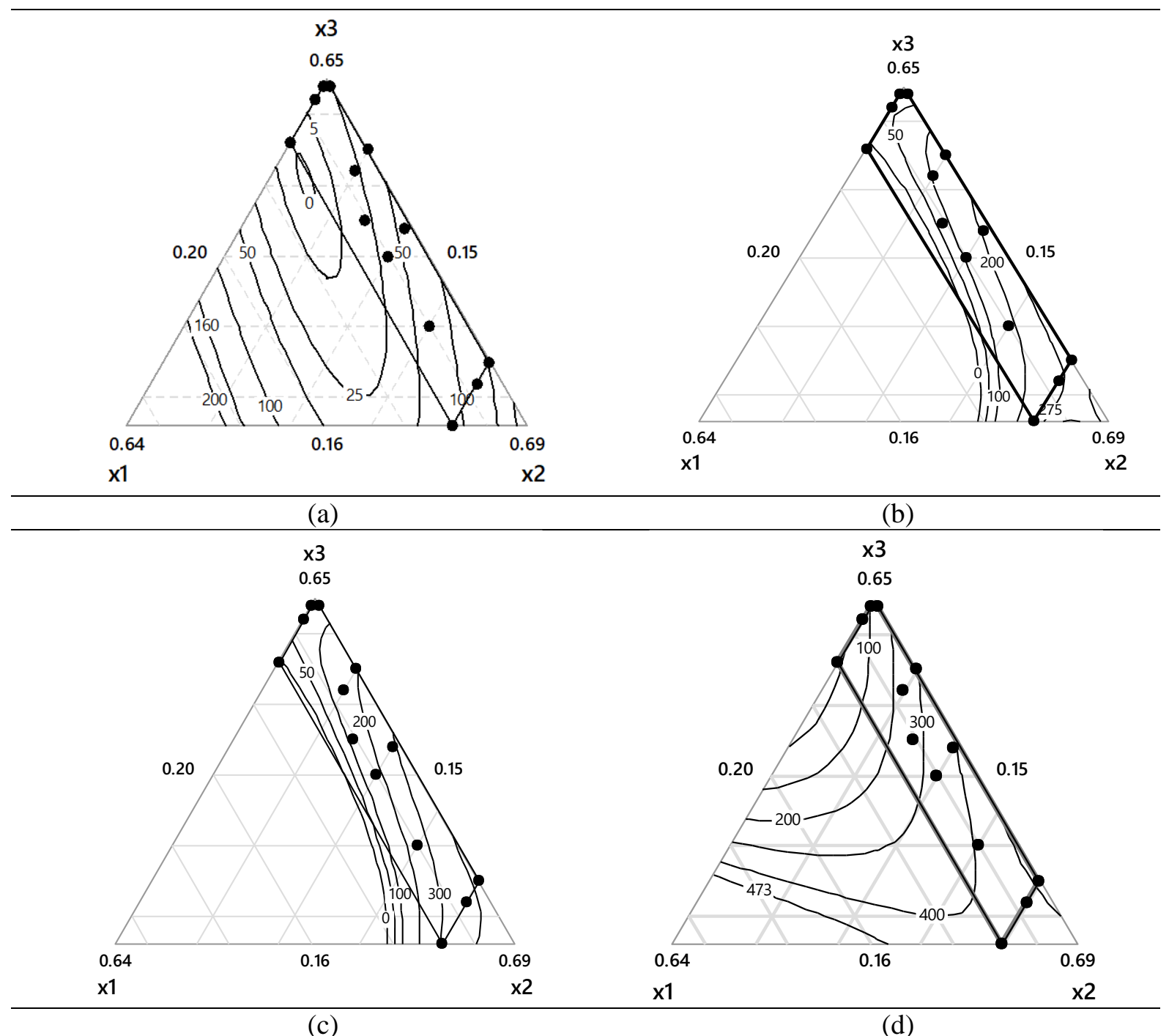




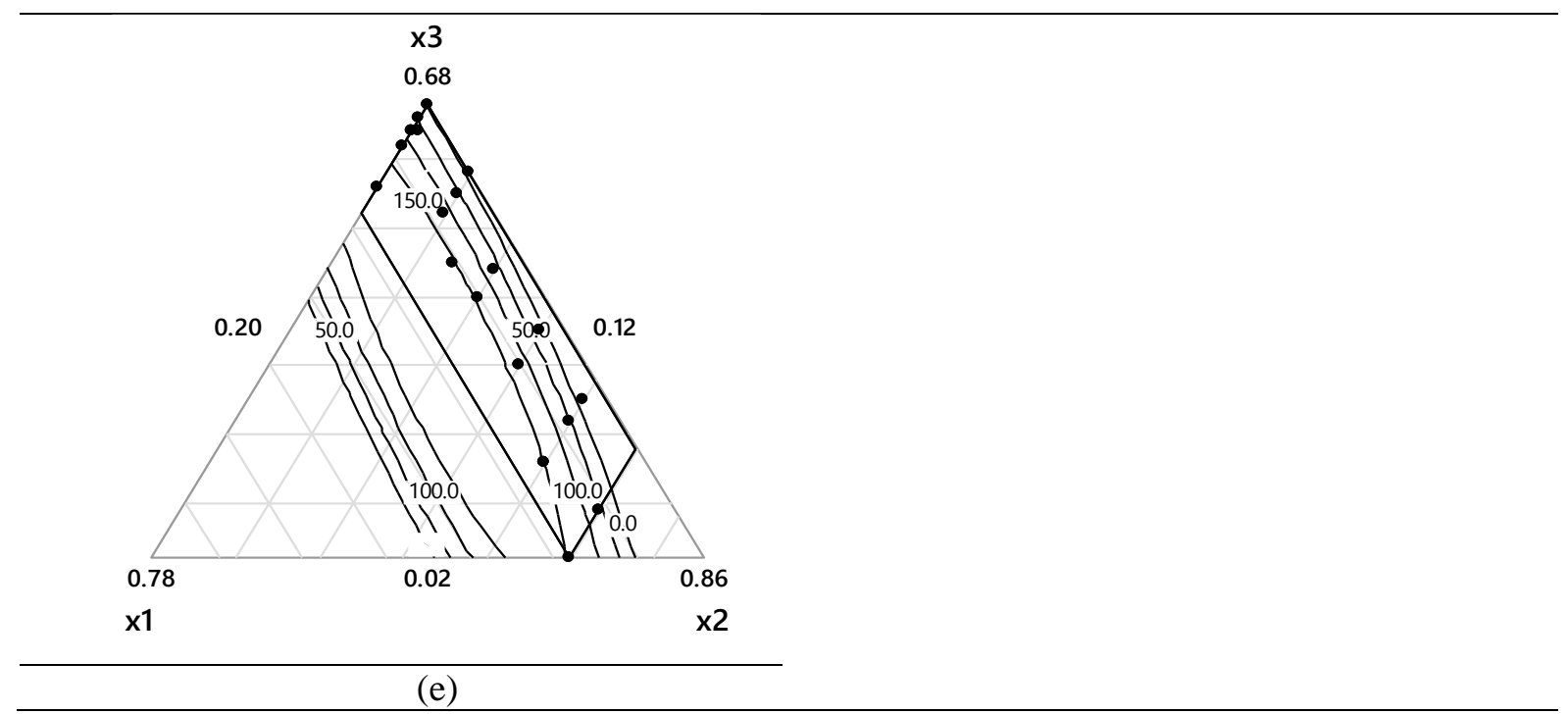

Figura 3. Gráficas de contorno de los modelos matemáticos ajustados y zona experimental: a) resistencia a la compresión a 24 h; b) resistencia a la compresión a 3 días; c) resistencia a la compresión a 7 días; d) resistencia a la compresión a 28 días; e) flujo de mortero.

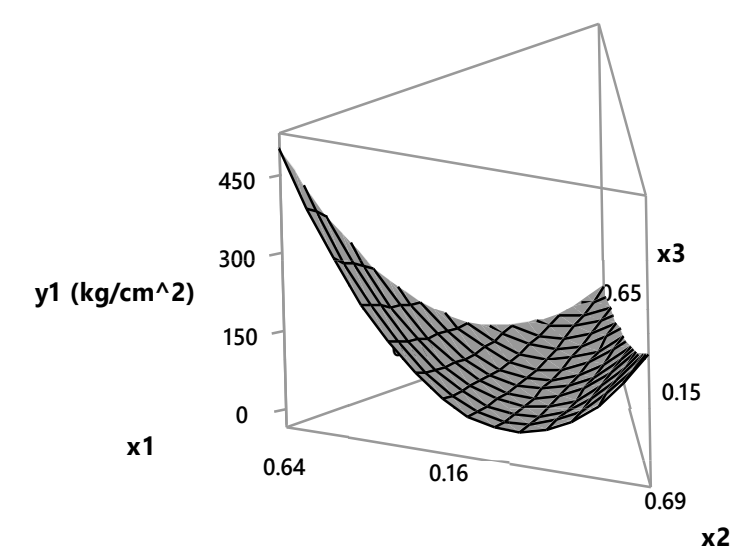

(a)

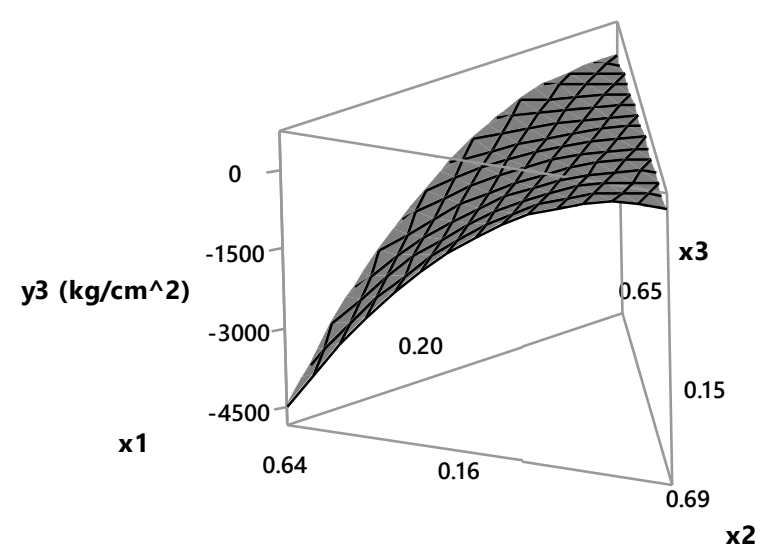

(c)

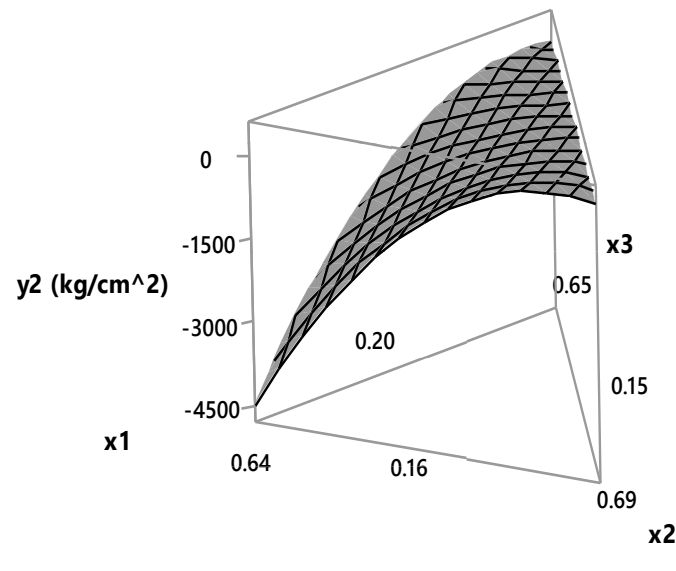

(b)

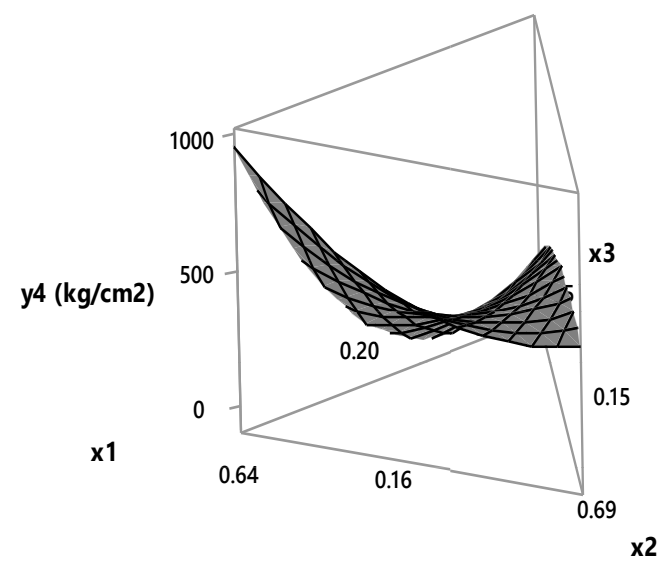

(d) 


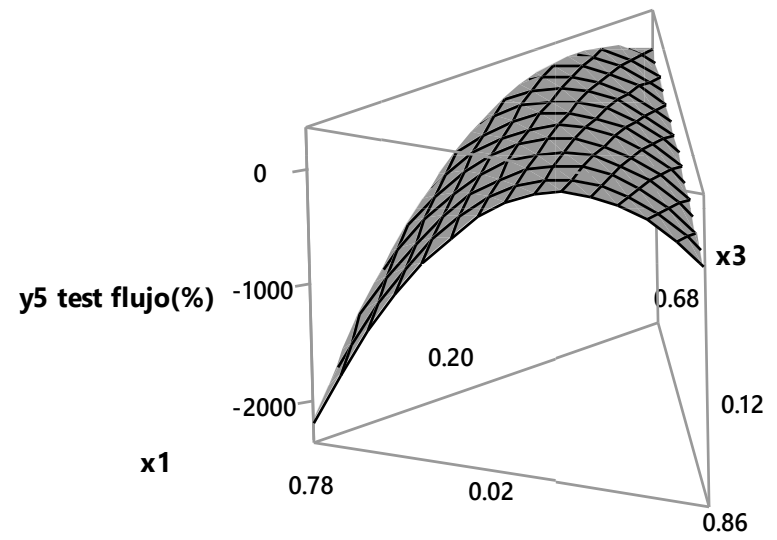

(e)

Figura 4. Superficies de respuesta de los modelos ajustados: a) resistencia a la compresión a las 24 h; b) resistencia a la compresión a los 3 días; c) resistencia a la compresión a los 7 días; d) resistencia a la compresión a los 28 días; e) flujo de mortero.

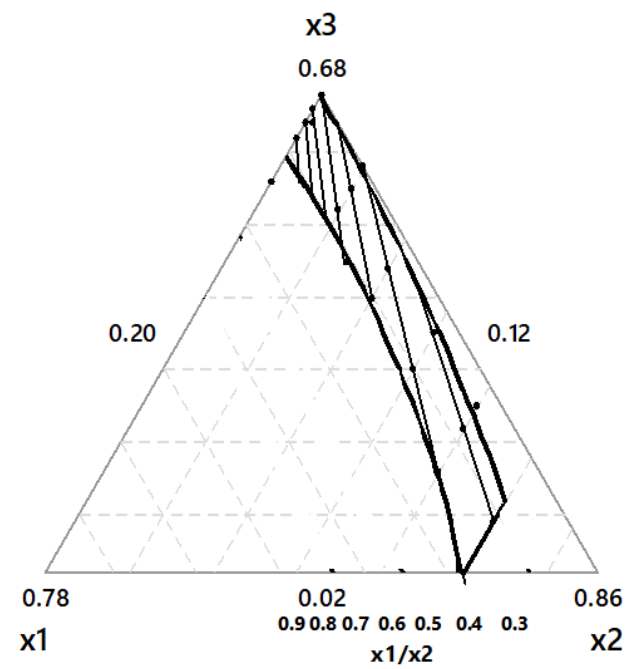

Figura 5. Zona de operabilidad en el simplex.

\subsection{Costos}

Se realizó una tabla de costo en función de los experimentos realizados (Tabla 10). En esta tabla se presenta el costo del mortero para un volumen de $1 \mathrm{~m}^{3}$, los flujos y la resistencia a los 28 días, también se muestra, como referencia para la aplicación que mejor convenga al constructor, también se puede visualizar las relaciones agua/cemento, las relaciones se encuentran en la zona de operabilidad y los experimentos que no han sido explorados. También pueden ser calculados, existe una tendencia marcada en los costos de esta muestra de experimentos las mezclas costosas se hallan en relaciones bajas y las más económicas en relaciones altas. Pero como ya se dijo antes, existen experimentos con las mismas relaciones, pero con comportamientos diferentes debido a las variaciones en sus proporciones, dando como resultados que los costos varíen, como el caso de la relación 0.4, para la cual los costos van desde 136.76 hasta $211.06 \$$, lo mismo ocurre con 0.3, para la cual los costos varían desde 202.99 hasta 229.73\$, y si se sigue explorando otras áreas dentro de la zona de operabilidad se hallarán un abanico más amplio de posibilidades. 
Tabla 10. Costos de los morteros en función de las fracciones de las mezclas.

\begin{tabular}{cccccccc}
\hline Experimento & $\mathrm{x}_{1}$ & $\mathrm{x}_{2}$ & $\mathrm{x}_{3}$ & $\mathrm{x}_{1} / \mathrm{x}_{2}$ & $\mathrm{y}_{4}\left(\mathrm{~kg} / \mathrm{cm}^{2}\right)$ & $\mathrm{y}_{5}$ test flujo $(\%)$ & Costo \$ para $1 \mathrm{~m}^{3}$ \\
\hline 7 & 0.18 & 0.60 & 0.22 & 0.30 & 473.35 & 45.15 & 202.99 \\
24 & 0.21 & 0.70 & 0.09 & 0.30 & 399.39 & 55.25 & 229.73 \\
8 & 0.16 & 0.40 & 0.44 & 0.40 & 438.90 & 58.73 & 136.76 \\
9 & 0.20 & 0.50 & 0.30 & 0.40 & 440.17 & 119.28 & 163.60 \\
3 & 0.24 & 0.60 & 0.16 & 0.40 & 411.60 & 148.40 & 188.28 \\
31 & 0.28 & 0.70 & 0.02 & 0.40 & 416.92 & 149.00 & 211.06 \\
10 & 0.15 & 0.30 & 0.55 & 0.50 & 306.67 & 42.70 & 103.23 \\
11 & 0.20 & 0.40 & 0.40 & 0.50 & 287.73 & 143.50 & 129.94 \\
6 & 0.20 & 0.35 & 0.45 & 0.59 & 243.77 & 155.00 & 11.46 \\
12 & 0.18 & 0.30 & 0.52 & 0.60 & 217.77 & 150.00 & 99.31 \\
45 & 0.14 & 0.20 & 0.66 & 0.70 & 150.03 & 28.08 & 69.41 \\
5 & 0.15 & 0.21 & 0.64 & 0.71 & 124.97 & 44.93 & 71.95 \\
4 & 0.16 & 0.20 & 0.64 & 0.80 & 106.10 & 78.60 & 67.63 \\
13 & 0.18 & 0.20 & 0.62 & 0.90 & 75.67 & 150.50 & 65.94 \\
\hline
\end{tabular}

\section{CONCLUSIONES}

Las relaciones agua/cemento y el diseño de vértices extremos permitieron delimitar una zona de operabilidad factible en el simplex para las mezclas de morteros, e incluso nos permite calcular, en función de las relaciones, nuevos experimentos en zonas no exploradas dentro de la delimitación. También las relaciones agua/cemento demostraron, dentro de esta metodología, que, aunque sean las mismas para un conjunto de mezclas, no significa que tengan la misma dosificación en sus componentes, por lo tanto, las mezclas no poseerán propiedades similares en sus repuestas. Como resultado, cada relación posee su rango de operabilidad experimental. Con la ayuda de esta metodología se logra encontrar proporciones adecuadas donde los componentes agua y cemento pueden interactuar formando pastas manejables. La importancia de este hallazgo no es trivial, ya que tanto como sucede a nivel macro puede ocurrir a nivel micro, o a nivel mineralógico, ya que las proporciones matemáticas se mantendrán.

Los modelos matemáticos se ajustaron con valores de $\mathrm{R}_{\mathrm{aj}}{ }^{2}$ mayores al $85 \%$ mostrando un alto nivel de explicación de las respuestas, así como valores p menores a 0.009 exponiendo que los modelos son significativos, el modelo que mejor comportamiento mostró fue el cuadrático, este fue el que no solamente se ajustó mejor al flujo sino también el modelo que mayor aportó para delimitar la zona de operabilidad. Esta metodología amplía la manera de hallar experimentos y permite, por lo tanto, encontrar mezclas cuyos costos se encuentren acordes a las necesidades técnicas del constructor, también, gracias a los modelos matemáticos, según sea el caso el constructor, se puede estimar las respuestas deseadas e incluso optimizarlas para cada necesidad.

\section{REFERENCIAS}

ACI COMMITTEE 211. (2002). Standard practice for slecting proportions for normal, heavy weight, and mass concrete. Farmington Hills, MI: American Concrete Institute, ACI Manual of Concrete Practice, pp. 1-91.

Akalin, O., Akay, K. U., Sennaroglu, B., Tez, M. (2010). Optimization of chemical admixture for concrete on mortar performance tests using mixture experiments. Chemometrics and Intelligent Laboratory Systems, 104(2), 233-242. https://doi.org/10.1016/j.chemolab.2010.08.013

Dinga, J.-T., Yan, P.-Y. L.-L., Zhu, J.-Q. (1999). Extreme vertices design of concrete with combined mineral admixtures. Cement and Concrete Research, 29(6), 957-960. https://doi.org/10.1016/S0008-8846(99)00069-1

Gutiérrez, H., De la Vara, R. (2008). Análisis y diseño de experimentos. México, D.F., México: McGraw-Hill/Interamericana Editores S.A. 
Mclean, R. A., Anderson, V. L. (1966). Extreme vertices design of mixture experiments. Technometrics, 8(3), 447-454.

Muñoz, E., Grau, M. (2013). Ingeniería química. Madrid: UNED.

Neville, A. M. (1999). Tecnologia del concreto. México, D.F., México: Addison Wesley Logman Ltd. Rivera, L. G. A. (2013). Concreto simple. Popayán, Colombia: Universidad del Cauca.

Sánchez de Guzmán, D. (2001). Tecnología del concreto y del mortero. Bogotá, Colombia: Pontificia Universidad Javeriana.

Simon, M. J., Lagergren, E. S., Snyder, K. A. (1997). Concrete mixture optimization using statistical mixture design methods. New Orleans, LA: International Symposium on High Performance Concrete, pp. 210-244.

Walpole, R., Myers, R., Myers, S., Ye, K. (2012). Probabilidad y estadística para ingeniería y ciencias. México DF, México: Pearson Education.

Yeh, I.-C. (2006). Analysis of strength of concrete using design of expeiments and neural networks. Journal of Materials in Civil Engineering, 18(4), 597-604. doi: 10.1061/_ASCE_0899-1 1561_2006_18:4(597) 